\title{
Caracterización de los recién nacidos con asfixia perinatal moderada o severa manejados con hipotermia cerebral selectiva en la Unidad de Recién Nacidos del Hospital Universitario San Ignacio desde junio de 2015 hasta marzo de 2017
}

Characterization of Newborns with Moderate or Severe Perinatal Asphyxia Managed with Selective Cerebral Hypothermia in the Newborn Unit of the San Ignacio University Hospital from June 2015 to March 2017

YARIS VARGAS-VACA Unidad de Cuidado Intensivo Neonatal, Departamento de Pediatría, Hospital Universitario San Ignacio Profesora del Departamento de Pediatría de la Facultad de Medicina, Pontificia Universidad Javeriana, Bogotá, Colombia

Claudia Devia Unidad de Cuidado Intensivo Neonatal, Departamento de Pediatría, Hospital Universitario San Ignacio Profesora ad honorem, Facultad de Medicina, Pontificia Universidad Javeriana, Bogotá, Colombia

\section{Ana María Bertolotto}

Unidad de Cuidado Intensivo Neonatal, Departamento de Pediatría, Hospital Universitario San Ignacio

Directora del Departamento de Pediatría, Facultad de Medicina, Pontificia Universidad Javeriana, Bogotá, Colombia

\section{Fernando SuÁrez-Obando a}

Servicio de Genética, Hospital Universitario San Ignacio

Instituto de Genética Humana, Facultad de Medicina, Pontificia Universidad Javeriana, Bogotá, Colombia

Director del Instituto de Genética Humana, Facultad de Medicina, Pontificia Universidad Javeriana, Bogotá, Colombia

\footnotetext{
${ }^{\text {a } C o r r e s p o n d e n c i a: ~ f e r n a n d o . s u a r e z @ j a v e r i a n a . e d u . c o ~}$
}

Recepción: 06/11/2018 | Aceptación: 05/06/2019

Cómo citar: Vargas-Vaca Y, Devia C, Bertolotto AM, Suárez-Obando F. Caracterización de los recién nacidos con asfixia perinatal moderada o severa manejados con hipotermia cerebral selectiva en la Unidad de Recién nacidos del Hospital Universitario San Ignacio desde junio de 2015 hasta marzo de 2017. Univ. Med. 2019;60(4). https:// doi.org/10.11144/Javeriana.umed60-4.crna

\section{RESUMEN}

Introducción: La encefalopatía hipóxico-isquémica altera el sistema nervioso central del recién nacido, con consecuencias que incluyen desde discapacidad física y mental hasta muerte. La moderada a severa debe manejarse con hipotermia terapéutica. Objetivo: Describir las características clínicas de un grupo de neonatos con encefalopatía tratados con hipotermia terapéutica en la Unidad de Cuidado Intensivo Neonatal del Hospital Universitario San Ignacio. Metodología: Estudio observacional de corte trasversal secuencial, desde junio de 2015 hasta marzo de 2017. Se compararon las variables continúas utilizando la $\mathrm{T}$ de Student. Se analizaron las frecuencias relativas y se compararon según variantes de interés clínico utilizando chi cuadrado. Resultados: El programa atendió 41 pacientes durante el periodo de observación. se evidenciaron convulsiones clínicas en el $65 \%$ de los sujetos. El principal desenlace clínico observado al egreso luego de la intervención fue algún grado de encefalopatía (90\%). Los determinantes clínicos del nacimiento del neonato se comparan con el desenlace muerte, donde no se encontraron diferencias significativas entre ambos grupos, a excepción de Apgar a los 10 minutos y acidemia. Para el desenlace muerte, RP mayores a 1 en el caso de cesárea, incremento de tiempo de coagulación, trombocitopenia, hipocalcemia y alteración de la función renal $(\mathrm{p}<0,05$ e IC-95\%: > 1). Conclusiones: El Apgar bajo a los 10 minutos y la acidemia presentaron mayor mortalidad. Hubo mayor prevalencia de alteraciones 
de coagulación, electrolíticas y renales en el grupo con desenlace letal. El tiempo de inicio de la terapia se relacionó con convulsiones, mas no con muerte. Palabras clave hipoxia; recién nacido; hipotermia; enfermedades del recién nacido; mortalidad perinatal.

Palabras clave

hipoxia; recién nacido; hipotermia; enfermedades del recién nacido; mortalidad perinatal.

\begin{abstract}
Introduction: hypoxic- ischemic encephalopathy generates various alterations in the central nervous system of the newborn, with various consequences ranging from physical and mental disability to death. The evidence indicates that moderate to severe hypoxic-ischemic encephalopathy must be managed with therapeutic hypothermia. Objectives: To describe the clinical characteristics of a group of neonates treated by the therapeutic hypothermia team of the Neonatal Intensive Care Unit of the Hospital Universitario San Ignacio. Methodology: Sequential cross-section observational study, from June 2015 to March 2017. Continuous variables were compared using student's t-test. Relative frequency analysis was performed and compared according to variants of clinical interest using chi-square. Results: A total of 41 patients were treated by the program during the observation period. Clinical seizures were evident in $65 \%$ of the subjects. The main clinical outcome to discharge observed in the patients after the intervention was encephalopathy (90\%). The clinical determinants of the birth of the neonate are compared with the death outcome where no significant differences were found proportionally between the two groups except for Apgar at 10 minutes and the presence of acidemia. For the outcome of death, RP greater than 1 in the case of Cesarean section, increased clotting time, thrombocytopenia, hypocalcemia and impaired renal function ( $p<0.05$ and IC-95> 1). Conclusions: Low Apgar at 10 minutes and acidemia is associated with higher mortality. There was a greater prevalence of electrolyte and renal coagulation alterations in the group with lethal outcome. The time of onset of the therapy was related to seizures but not to death.

Keywords

hypoxia; newborn; hypothermia; infant; newborn; diseases; perinatal mortality.
\end{abstract}

\section{Introducción}

La asfixia perinatal constituye una de las principales causas de mortalidad en niños menores de cinco años de edad $(1,2)$. Se estima que por cada paciente que fallece por asfixia perinatal hay 10 sobrevivientes con secuelas graves (3). En el Distrito Capital, para 2014 la asfixia perinatal representó el
$6,4 \%$ de la mortalidad neonatal temprana (4). La encefalopatía hipóxico-isquémica (EHI) es el conjunto de alteraciones en el sistema nervioso central del recién nacido, generado por la privación de oxígeno y la isquemia subsiguiente. Originalmente, este cuadro clínico lo describieron y clasificaron Sarnat y Sarnat $(5,6)$.

Desde 2010, la Asociación Americana del Corazón y el Comité Internacional en Resucitación han recomendado que a todo recién nacido mayor de 36 semanas de edad gestacional que presente una EHI de moderada a severa se le ofrezca la posibilidad de hipotermia terapéutica dentro del manejo posterior a la reanimación. La misma recomendación fue avalada en la guía de práctica clínica del recién nacido con asfixia perinatal, publicada en abril de 2013 por el Ministerio de Salud y Protección Social de Colombia $(7,8)$.

Los hallazgos actuales han demostrado que el uso de hipotermia terapéutica dentro de las primeras seis horas posteriores al evento asfíctico reduce estadística y clínicamente el resultado combinado de mortalidad o discapacidad superior a los 18 meses, sin aumento del riesgo de discapacidad entre los sobrevivientes y con efectos positivos que se mantienen hasta la infancia $(9,10)$.

El programa de Hipotermia Selectiva con Cool-Cap (casco) en la Unidad de Cuidado Intensivo Neonatal del Hospital Universitario San Ignacio ofrece esta opción terapéutica a los recién nacidos que presentan EHI. El presente artículo describe la experiencia del equipo tratante con un grupo de neonatos atendido en el hospital. Ello aporta al conocimiento de esta área de la atención del neonato, al presentar desenlaces clínicos de interés en el contexto de un hospital de alta complejidad en Colombia.

En Colombia ya se describió una cohorte de pacientes tratados con hipotermia terapéutica corporal total durante 2017 (11). El estudio pretendía describir los resultados obtenidos con el otro método de hipotermia: manta 


\section{Métodos}

Estudio observacional de corte trasversal secuencial. Los datos de los pacientes comprenden una observación desde junio de 2015 a marzo de 2017. El tamaño de la muestra fue censal, e incluyó a todos los pacientes referidos al programa durante el periodo de observación con criterio de asfixia, definida por la Academia Americana de Pediatría y el Colegio Americano de Ginecología y Obstetricia como acidosis metabólica o mixta ( $\mathrm{pH}$ menor de 7,0 en una muestra de sangre de cordón al nacer), Apgar menor de 3 a los 5 minutos, afectación neurológica (convulsiones, coma e hipotonía) y afectación de múltiples órganos (corazón, pulmón, hígado, riñón e intestinos) (6).

La terapia de hipotermia cerebral selectiva con el sistema Cool-Cap se utiliza para tratar a recién nacidos con EHI de moderada a severa, a fin de enfriar selectivamente la cabeza con una moderada hipotermia sistémica ( 34 a $\left.35^{\circ} \mathrm{C}\right)$, de reducir la mortalidad y de prevenir o reducir la severidad del daño neurológico asociado con la EHI. Los pacientes permanecen en cuidado intensivo con monitorización continua de signos vitales y de actividad cerebral mediante electroencefalograma ampliado. La terapia tiene una duración de 72 horas, posterior a la cual se realiza el recalentamiento a $0,5^{\circ} \mathrm{C}$ por hora hasta alcanzar las temperaturas fisiológicas (36,5 a 37,5 $\left.{ }^{\circ} \mathrm{C}\right)$.

Las variables que se estudiaron se definieron según lo reportado en la literatura $(5,12)$ y el protocolo de atención de la unidad de recién nacidos. Las variables se recolectaron directamente de la historia clínica de los pacientes y se tabularon en una hoja de cálculo en Excel ${ }^{\circledR} 2010$ y exportadas al software Stata 14 para su análisis. Se consideraron variables de desenlace de la atención la presencia de encefalopatía al egreso y muerte. Las gráficas se generaron con GraphPad 7.04.

Se compararon las variables continuas utilizando la T de Student. Se analizaron las frecuencias relativas y se compararon de acuerdo con variantes de interés clínico utilizando chi cuadrado. También se calcularon la razón de prevalencias y sus intervalos de confianza al 95\% (IC-95\%) para los desenlaces de encefalopatía y muerte según las variables clínicas de interés. Se consideró un $\alpha=0,05$ ( $p$ significativa $<0,05)$ para todos los estadísticos utilizados. Para las variables categóricas como el Apgar se clarificaron tres grupos: 0-5, 6-7 y 8-10, y se compararon utilizando Kruskal-Wallis.

Las variables se definieron de la siguiente manera: arritmia grave (arritmia diferente a bradicardia sinusal o bigeminismo), hipotensión grave (cuando a pesar de la administración de volumen se requirió dopamina a más de $20 \mu \mathrm{g} /$ $\mathrm{kg}$ cada minuto) y trombosis venosa (trombosis de vasos no relacionada con líneas de acceso venoso). Para las demás variables definidas como complicaciones posnatales se tomó un tiempo de 7 días de vida, y se consideró:

Hipotensión leve: tensión arterial media menor de $40 \mathrm{~mm} \mathrm{Hg}$.

Coagulopatía: sangrado clínico o alteración de los tiempos de coagulación.

Falla renal: gasto urinario $<0,5 \mathrm{ml} / \mathrm{kg}$ cada hora durante más de 24 horas o aumento de la creatinina más de $009 \mathrm{mmol} / \mathrm{l}$

Hiponatremia: sodio sérico $<135 \mathrm{mmol} / \mathrm{l}$,

Hipocalemia: potasio sérico $<3,5 \mathrm{mmol} / 1$

Trombocitopenia: recuento de plaquetas < 150.000 por $\mathrm{uL}$.

Elevación de enzimas hepáticas: AST > 200 $\mathrm{IU} / \mathrm{y}$ y ALT > 100I U/l.

Acidosis metabólica después de entrar en el protocolo: $\mathrm{pH}<7,34$ o déficit de base $\geq 4 \mathrm{mmol} /$ 1.

Dificultad respiratoria: necesidad de ventilación mecánica o presión positiva continua en la vía aérea (CPAP)

Infección sistémica: cultivos positivos en sangre, líquido cefalorraquídeo o en orina tomadas después de ingresar a la terapia.

Hemoconcentración: aumento del hematocrito $>20 \%$.

Hipoglucemia: glucemia $<47 \mathrm{~m} / \mathrm{dl}$.

Hipocalcemia: calcio $<8 \mathrm{mmol} / \mathrm{l}$.

Dificultad para mantener la temperatura objetivo: menos de $33,5^{\circ} \mathrm{C}$ o más de $37,5^{\circ} \mathrm{C}$ por más de una hora. 


\section{Aspectos éticos}

Todos los datos se reportan de manera agregada y anónima. La base de datos reposa en los computadores de los investigadores bajo usuario y contraseña únicas y bajo los parámetros de seguridad de la Pontificia Universidad Javeriana. El protocolo de estudio fue revisado y aprobado por el Comité de Ética de la Investigación de la Facultad de Medicina y el Hospital Universitario San Ignacio.

\section{Resultados}

El programa atendió 41 pacientes durante el periodo de observación. Se consideraron criterios de ingreso al protocolo los recién nacidos mayores o iguales a 36 semanas de edad gestacional y con un peso mayor o igual a 1800 gramos, que cursaban con encefalopatía moderada o severa definidos por criterios A y B, indicados como:

Criterios A: uno o más de los siguientes hallazgos: calificación de Apgar menor o igual a 5 a los 10 minutos, reanimación continua incluida intubación endotraqueal o máscara 10 minutos después del nacimiento, $\mathrm{pH}$ de sangre de cordón umbilical menor a 7,0 en la primera hora de vida, base exceso mayor o igual a -16 en gases de cordón umbilical o cualquier muestra de sangre en los primeros 60 minutos de vida.

Criterio B: alteración del estado de conciencia (letargo, estupor o coma) y, al menos, uno de los siguientes: hipotonía, reflejos anormales o anormalidades pupilares, ausencia de succión o débil, convulsiones clínicas.

Si el paciente cumplía con los criterios A y $B$, se realizaba electroencefalograma de amplitud. Se excluyeron pacientes con ano imperforado y anormalidades congénitas mayores. Se incluyó un paciente con panhipopituitarismo, quien al momento del nacimiento no contaba con este diagnóstico.

Las características generales de ingreso de los sujetos al programa se describen en la tabla 1 . El $14 \%$ de los casos tenía entre 36 y 37 semanas de edad gestacional (EG), el $82 \%$ de los casos tenía entre 38 y 40 semanas de EG, un solo caso fue de
41 semanas. La edad gestacional más frecuente fue 39 semanas $(41,5 \%)$. La vía de nacimiento más frecuente fue el parto $(51,2 \%)$. En relación con la actividad electroencefalográfica (EEG) antes de la intervención, el $61 \%$ de los casos padecía de actividad anormal de tipo moderado y el $17 \%$ tenía actividad EEG anormal del tipo severa.

\section{Tabla 1}

Características generales de los pacientes atendidos por el programa

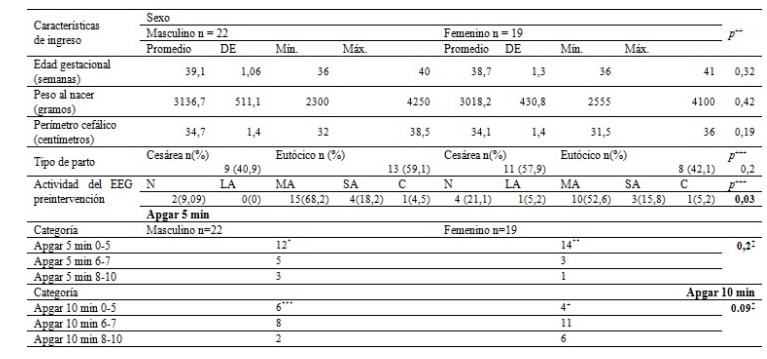

Las principales características que definen el parto se presentan discriminadas por sexo. No se encontraron diferencias significativas en la proporción de casos por sexo relacionados con edad gestacional, peso al nacer, perímetro cefálico Apgar o tipo de parto. Predominó la actividad electroencefalográfica anormal de tipo moderado presente en el $61 \%$ de los casos. *Basado en 20 observaciones. ${ }^{* *}$ Basado en 18 observaciones **** Basado en 19 observaciones ${ }^{+}$Basado en 17 observaciones. DE: Desviación estándar. $\mathrm{N}$ : normal. LA: leve anormal. MA: moderado anormal. SA: severo anormal. C: convulsivo.

${ }^{+}$Diferencias de medias con varianzas iguales. ${ }^{+}$

++ Diferencia de proporciones. ${ }^{\ddagger}$ Kruskal-Wallis.

El tratamiento se realizó durante 72 horas. Las principales complicaciones posnatales se aprecian en la tabla 2. Las convulsiones clínicas se evidenciaron en el $65 \%$ de los sujetos. La dificultad respiratoria se apreció en el $48 \%$ de los pacientes. En relación con los trastornos electrolíticos, la hiponatremia fue el más frecuente, en el 56\% de los casos; la hipocalemia, en el 31\%, y la hipocalcemia, en el 26\% de los pacientes. La hipertensión pulmonar se presentó en el 29\% de los pacientes y los trastornos de coagulación se evidenciaron en el 26\% de los neonatos. Es frecuente observar bradicardia sin repercusión hemodinámica durante la terapia. 
No se presentó ningún caso de trombosis venosa ni hemoconcentración.

\section{Tabla 2}

Eventos adversos durante la terapia

\begin{tabular}{|c|c|c|c|c|c|}
\hline & \multicolumn{2}{|c|}{$\begin{array}{c}\text { Efectos adversos } \\
\text { mayores }\end{array}$} & \multirow[b]{2}{*}{ Sin (\%) } & \multirow[b]{2}{*}{ Non $(\%)$} & \\
\hline & Sín (\%) & Non $(\%)$ & & & \\
\hline Hipotensión intratable & $3(13,6)$ & $19(86,7)$ & $3(15,8)$ & $16(84,2)$ & 1 \\
\hline Trombosis venosa & $O(0)$ & $22(100)$ & $O(0)$ & $19(100)$ & \\
\hline Hipotensión grave & $3(13,6)$ & $19(86,7)$ & $4(21)$ & $15(79)$ & 0,6 \\
\hline \multicolumn{6}{|l|}{ Complicaciones posnatales } \\
\hline \multicolumn{6}{|l|}{ Hipotensión leve } \\
\hline Coagulopatia & $1(4,5)$ & $21(95,5)$ & $3(15,8)$ & $16(84,2)$ & 0,3 \\
\hline Incremento TC & $6(27,3)$ & $16(72,7)$ & $5(26,3)$ & $14(73,7)$ & 1 \\
\hline Trombocitopenia & $2(9,1)$ & $20(90,9)$ & $1(5,2)$ & $18(94,8)$ & 1 \\
\hline Hemoconcentración & $O(0)$ & $22(100)$ & $O(0)$ & $19(100)$ & 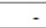 \\
\hline Arritmia leve & $O(0)$ & $22(100)$ & $O(0)$ & $19(100)$ & - \\
\hline Hipocalemia & $8(40)$ & $12(60)^{*}$ & $5(26,3)$ & $14(73,7)^{*}$ & 0,3 \\
\hline Hiponatremia & $7(35)$ & $13(65)^{*}$ & $16(72,3)$ & $3(27,3)$ & 0,2 \\
\hline Hipocalcemia & $7(35)$ & $13(65)^{*}$ & $4(21,1)$ & $15(78,9)$ & 0,7 \\
\hline Hipoglicemia & $4(19,1)$ & $17(80,9)$ & $6(31,6)$ & $13(68,4)$ & 0,4 \\
\hline Alteración FR & $1(4,5)$ & $21(95,4)$ & $3(13,6)$ & $16(84,2)$ & 0,3 \\
\hline Falla renal & $1(4,5)$ & $21(95,4)$ & $1(5,2)$ & $18(94,8)$ & 1 \\
\hline Acidosis metabólica & $2(9,1)$ & $20(90,9)$ & $1(5,2)$ & $18(94,8)$ & 1 \\
\hline Dificultad respiratoria & $5(22,7)$ & $17(77,3)$ & $15(79)$ & $4(21)$ & 1 \\
\hline Hipertensión pulmonar & $8(38)$ & $13(62)$ & $4(21,1)$ & $15(78,9)$ & 0,3 \\
\hline Incremento de TA & $6(35,3)$ & $11(64,3)^{+}$ & $3(18,8)$, & $13(81,2)^{++}$ & 0,4 \\
\hline Infección sistémica & $1(4,5)$ & $21(95,5)$ & $1(5,2)$ & $18(94,8)$ & 1 \\
\hline Sepsis & $3(13,6)$ & $19(86,7)$ & $0(0)$ & $19(100)$ & 0,3 \\
\hline Encefalopatía & $19(86,7)$ & $3(13,6)$ & $18(94,8)$ & $1(5,2)$ & 0,6 \\
\hline Convulsión clínica & $15(68,2)$ & $7(31,8)$ & $12(63,2)$ & $7(36,8)$ & 0,7 \\
\hline Videotelemetría anormal & $4(26,7)$ & $\begin{aligned} 11(73,3) \\
+++\end{aligned}$ & $7(46,7)$ & $8(53,3)^{+++}$ & 0,1 \\
\hline
\end{tabular}

Proporción de pacientes por tipo de complicación.

TC: tiempo de coagulación. FR: función renal.

TA: Transaminasas. *Porcentaje sobre un total de 20 pacientes. **Porcentajes basados en un total de 18 pacientes. + Porcentajes basados en 17 pacientes. ++ Porcentajes basados en 16 pacientes. +++ Anormal/Normal. Porcentajes basados en 15 pacientes. $¥$ Test exacto de Fisher.

El principal desenlace clínico observado al egreso de los pacientes luego de la intervención fue la encefalopatía (90\%), determinada con base en tres criterios: la videotelemetría; resonancia magnética cerebral tomada a los 3 y los 5 días, respectivamente, después de terminada la terapia, y el examen clínico realizado por neurología pediátrica previo al egreso. Se consideró algún grado de encefalopatía si existía anormalidad en uno o más criterios. Nuestro estudio realizó seguimiento desde el ingreso a la terapia hasta el alta. Del total de pacientes, el $63,3 \%$ tenía una videotelemetría anormal, y el $51 \%$ de los pacientes, una resonancia anormal. Al comparar las complicaciones luego de la intervención en relación con el promedio de tiempo desde el nacimiento hasta el inicio de la hipotermia, solo se presentó una diferencia significativa en el inicio tardío de la intervención con la aparición de convulsiones clínicas. Vale la pena aclarar que solo 5 pacientes recibieron la terapia en las primeras 6 horas (tabla 3).

Tabla 3

Complicaciones del tratamiento en relación con el tiempo de inicio de la atención

\begin{tabular}{|l|r|r|r|r|r|}
\hline \multirow{2}{*}{ Complicación del tratamiento } & \multicolumn{4}{|c|}{ Afectados } & \multirow{2}{*}{$\boldsymbol{*}^{*}$} \\
\cline { 2 - 5 } & \multicolumn{2}{|c|}{ Presente } & \multicolumn{2}{c|}{ Ausente } \\
\cline { 2 - 5 } & $\boldsymbol{\mu}$ (tiempo) & $\boldsymbol{N}$ & $\boldsymbol{\mu}$ (tiempo) & \\
\hline Arritmia leve & - & - & 41 & 11 & - \\
\hline Arritmia grave & 1 & 6 & 40 & 11,1 & - \\
\hline Hipotensión leve & 12 & 11,7 & 29 & 10,7 & 0,5 \\
\hline Hipotensión grave & 7 & 7,8 & 37 & 11,6 & 0,06 \\
\hline Hipotensión intratable & 6 & 11,5 & 35 & 8 & 0,1 \\
\hline Coagulopatia & 4 & 10,7 & 37 & 10,7 & 0,2 \\
\hline Incremento TC & 11 & 9,6 & 29 & 11,6 & 0,2 \\
\hline Trombocitopenia & 3 & 12,7 & 38 & 10,8 & 0,5 \\
\hline Hemoconcentración & - & - & 41 & 11 & - \\
\hline Trombosis venosa & - & - & 41 & 11 & - \\
\hline Hipocalemia & 13 & 12,1 & 26 & 11 & 0,5 \\
\hline Hiponatremia & 10 & 9,3 & 29 & 12 & 0,1 \\
\hline Hipocalcemia & 10 & 9 & 29 & 12,1 & 0,06 \\
\hline Hipoglucemia & 10 & 11,3 & 30 & 11 & 0,8 \\
\hline Alteración FR & 4 & 12 & 36 & 11 & 0,7 \\
\hline Falla renal & 2 & 11,5 & 39 & 11 & 0,8 \\
\hline Acidosis metabolica & 38 & 10,8 & 3 & 13,3 & 0,3 \\
\hline Dificultad respiratoria & 32 & 11 & 9 & 10,9 & 0,9 \\
\hline Hipertensión pulmonar & 12 & 11,4 & 28 & 11 & 0,8 \\
\hline Incremento de TA & 9 & 12,3 & 24 & 11,5 & 0,6 \\
\hline Infección sistémica & 2 & 6,5 & 39 & 12,2 & 0,1 \\
\hline Sepsis & 2 & 11,6 & 38 & 10,9 & 0,8 \\
\hline Encefalopatia & 37 & 11 & 4 & 10,5 & 0,8 \\
\hline Convulsión clínica & 27 & $\mathbf{1 2 , 3}$ & 14 & $\mathbf{8 , 2}$ & $\mathbf{0}, 009$ \\
\hline Videotelemetría anormal & 12 & 11,5 & 18 & 11,6 & 0,9 \\
\hline
\end{tabular}

Nota: se comparan las complicaciones presentadas luego de la intervención, en relación con el promedio de tiempo en que se inició la hipotermia (promedio de número de horas desde el nacimiento hasta el inicio de la intervención). El promedio de horas de inicio de la atención fue significativamente mayor en el caso de pacientes con convulsión clínica.

TC: tiempo de coagulación; FR: función renal. TA: Transaminasas. *T de Student.

Las figuras 1 y 2 permiten comparar los desenlaces de supervivencia o muerte luego de la intervención en relación con las mismas variables clínicas de interés de cada neonato. Los pacientes con Apgar más bajo a los 10 minutos y la acidemia presentaron mayor mortalidad, la cual correspondió al $17 \%$. Todos los pacientes que fallecieron tenían gases en la sangre del cordón < 6,91 y base exceso mayor de -19 . Un $14 \%$ de los pacientes que ingresaron a la terapia tenían $\mathrm{pH}$ en la sangre del cordón umbilical igual a 7,0 y un $17 \%$ mayor a 7,0 . 


\section{Figura 1}

Eventos de encefalopatía relacionados con variables clínicas de interés

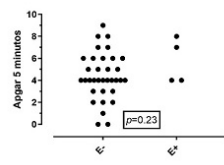

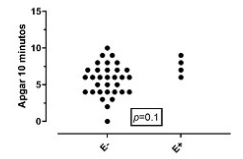
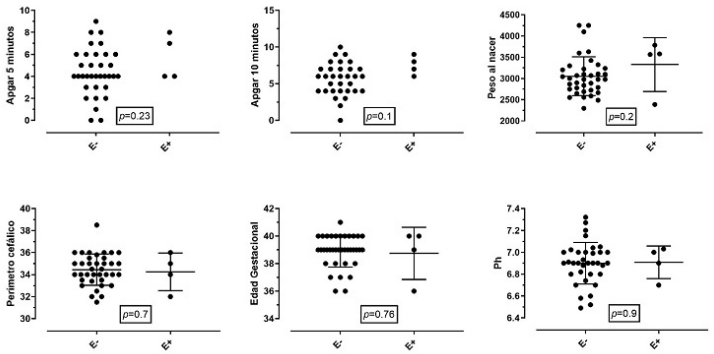

Nota: los determinantes clínicos del nacimiento del neonato se comparan con la presencia de encefalopatía. No se encontró que se presentara, proporcionalmente, diferencias significativas entre ambos grupos. Peso al nacer en gramos. Perímetro cefálico en centímetros. Edad gestacional en semanas. E+: encefalopatía presente. E-: encefalopatía ausente. La gráfica de Apgar solo muestra los eventos, sin media ni la DE.

\section{Figura 2}

Desenlace de los pacientes (vivo o muerto)
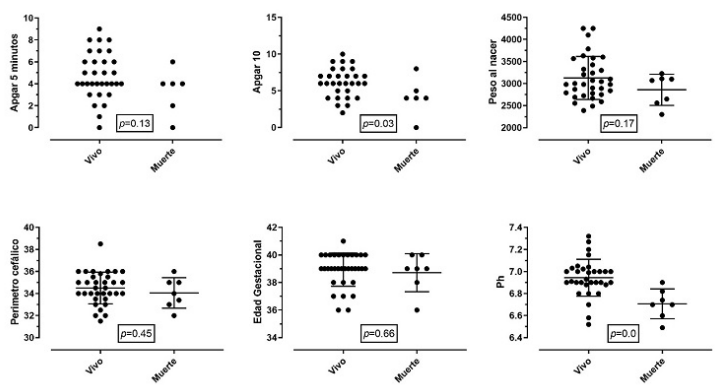

Nota: Los determinantes clínicos del nacimiento del neonato se comparan con el desenlace de muerte. No se encontró que se presentara, proporcionalmente, diferencias significativas entre ambos grupos a excepción de Apgar a los

10 minutos y presencia de acidemia. Peso al nacer en gramos. Perímetro cefálico en centímetros.

Edad gestacional en semanas. La gráfica de Apgar solo muestra los eventos, sin media ni la DE.

Los desenlaces de mortalidad y encefalopatía se compararon en relación con el tiempo de atención desde el momento de nacimiento hasta el inicio de la intervención. Cada uno de los eventos se aprecia en la figura 3.
Figura 3

Tiempo de atención en relación con la mortalidad
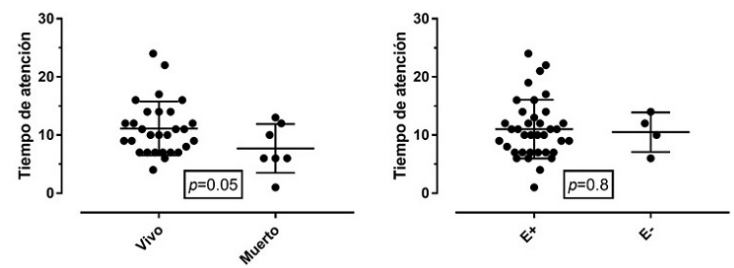

Comparación del promedio de tiempo para el inicio de la intervención luego del nacimiento en relación con los desenlaces de mortalidad y presencia de encefalopatía. E+: encefalopatía presente. E-: encefalopatía ausente.

En la tabla 4 se aprecia la comparación de la razón de prevalencias (RP) de encefalopatía y muerte de acuerdo con exposiciones de interés clínico. En cuanto al desenlace de encefalopatía se evidenciaron RP mayores a 1, en los casos de acidosis metabólica, dificultad respiratoria, hipertensión pulmonar y convulsiones clínicas. Sin embargo, los IC-95\% pasan por un RP de 1. Para el desenlace de muerte, se aprecian RP mayores a 1 en el caso de cesárea, incremento de tiempo de coagulación, trombocitopenia, hipocalcemia y alteración de la función renal ( $\mathrm{p}$ $<0,05$ e IC-95\% mayores a 1 ).

\section{Tabla 4}

Razón de prevalencias entre la exposición de variables de interés clínico y los desenlaces de encefalopatía y muerte

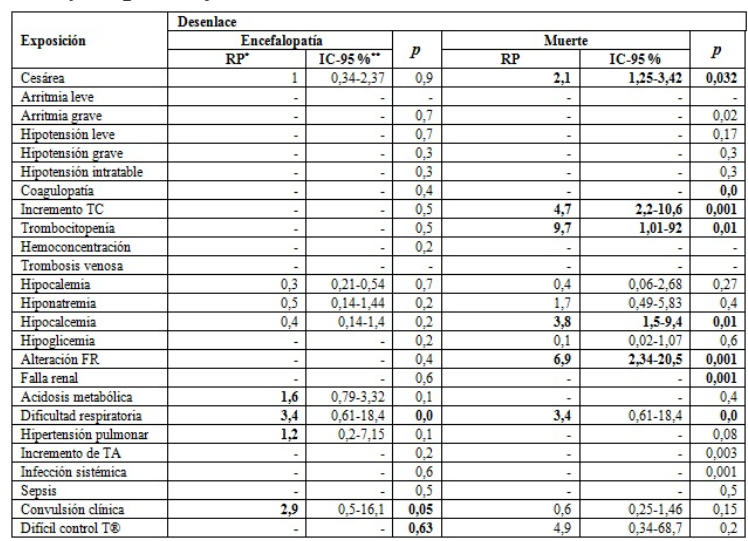

RP: razón de prevalencias. IC-95\%: intervalo de confianza al 95\%. FR: función renal. TA: transaminasas. 
Un paciente tenía unos gases iniciales compatibles con asfixia, por lo que ingresó al protocolo, pero posteriormente se determinó que tenía panhipotuitarismo.

\section{Discusión}

De acuerdo con los datos suministrados por el Área de Vigilancia en Salud Pública de la Secretaría Distrital de Salud, basados en registros del Registro Único de Afiliados, la tasa de mortalidad perinatal en Bogotá ha mostrado un descenso importante, de hasta 13,4 por cada 1000 nacimientos para 2017. La mortalidad neonatal temprana se había mantenido alrededor de 5,9 por cada 1000 nacimientos hasta el 2013 y comenzó a caer hasta lograr un 4,3 por cada 1000 nacimientos para 2014 (13). Estas tendencias se asemejan a la disminución de la mortalidad tanto en países en desarrollo $(14,15)$ y economías emergentes (16) como en países desarrollados $(17,18)$. Esta reducción se debe al control de patologías infecciosas perinatales (19) y al mejor manejo de las complicaciones derivadas del parto (20) y otras patologías del neonato, incluidas malformaciones congénitas (21), así como la mejoría en las técnicas de reanimación (22).

El Hospital Universitario San Ignacio hace parte de la Red Distrital de Neuroprotección desde 2015 y ofrece un manejo de probada eficacia clínica (23) a los neonatos con asfixia moderada o severa referidos de diferentes instituciones de salud del Distrito y del departamento, independiente de su seguridad social.

En la tabla 1 se aprecia que la actividad del EEG antes de la intervención tiene de manera significativa resultados moderada y severamente anormales. La actividad del EEG es un marcador sensible de daño cerebral tanto en neonatos pretérmino (24) como a término (25). La encefalopatía es un síndrome progresivo, por lo que esta herramienta cada día cobra más importancia y nos ayuda a determinar si un paciente se beneficia de entrar al protocolo reportado en la literatura con un VPP del 75\% para un evento adverso, y la combinación de este con un examen alterado tiene un VPP superior que cada uno por separado (26).

Varias alteraciones clínicas se producen tanto por la patología de base como por la terapia y no pueden ser discriminadas con precisión (27). Sin embargo, en nuestro estudio, algunas de ellas fueron predictoras de mortalidad como el Apgar bajo a los 10 minutos, la acidemia, el antecedente de cesárea, el incremento de los tiempos de coagulación, la trombocitopenia, la hipocalcemia y la alteración de la función renal (27).

El $90 \%$ de los pacientes se diagnosticó con algún grado de encefalopatía al egreso, determinada por alguna alteración encontrada en la videotelemetría, la resonancia magnética cerebral o el examen físico neurológico. Sin embargo, de los pacientes que sobrevivieron solo un paciente requirió gastrostomía y fue diagnosticado con panhipopituitarismo; los demás egresaron con succión adecuada. El $41,46 \%$ de los pacientes fue dado de alta con encefalopatía leve.

No fue posible un seguimiento a más largo plazo a nuestros pacientes, porque las aseguradoras de salud no los derivan a nuestra institución para controlarlos. A diferencia del estudio de Manotas et al. (11), de hipotermia corporal total, la mortalidad de nuestros pacientes no se relacionó con el tiempo de inicio de la terapia. Todos los pacientes que fallecieron en nuestro estudio tenían $\mathrm{pH}$ en gases de sangre de cordón $<6,91$ y base exceso mayor de -19 , 6 de ellos con pH menor de 6,7. Compartimos la trombocitopenia como factor de riesgo de mortalidad cuando se encuentra por debajo de 100.000.

Los pacientes que más tardaron en entrar a la terapia de hipotermia activa presentaron más convulsiones y encefalopatía; sin embargo, para mortalidad, fue similar el tiempo promedio de atención en ambos tipos de desenlace. Este hallazgo podría considerarse de importancia, para apoyar la entrada de pacientes a hipotermia aun después de las 6 horas de ventana terapéutica descritas inicialmente para el protocolo, aunque con mayor riesgo de presentar convulsiones clínicas. Estudios recientes apoyan esta hipótesis (28). 
Un 14\% de los pacientes que ingresó a la terapia tenía $\mathrm{pH}$ en sangre de cordón umbilical igual a 7 , y un $17 \%$, mayor a 7 ; sin embargo, fueron ingresados a la terapia por cumplir criterios A y B, lo que lleva a concluir que no es el único ni más importante criterio para definir los pacientes que requieren la terapia, pero si se considera un predictor de mortalidad, porque ninguno de estos pacientes falleció. Algunos pacientes tienen diagnósticos diferenciales que deben ser tenidos en cuenta y que pueden simular estados de asfixia, como ocurrió con nuestro paciente con panhipotuitarismo.

\section{Conclusiones}

Los pacientes que tuvieron Apgar bajo a los 10 minutos y acidemia severa presentaron mayor mortalidad. Todos los pacientes que fallecieron tenían gases en sangre de cordón $<6,91$ y base exceso mayor de -19 . Se evidenció una mayor prevalencia de alteraciones de coagulación, electrolíticas y renales en el grupo con desenlace letal.

Con base en los resultados encontrados, es prioritario definir con mayor precisión el proceso de remisión de los pacientes en tiempo óptimo y contar a futuro con la clínica de asfixia que nos permita garantizar el seguimiento integral y multidisciplinario de los pacientes manejados con esta terapia. Sin embargo, en nuestro estudio no se encontró una diferencia importante en mortalidad cuando la terapia se inició luego de seis horas.

Dada la diferencia en mortalidad encontrada, comparada con la de hipotermia corporal, se requieren estudios adicionales para determinar si en nuestro medio las dos terapias son igualmente efectivas.

\section{Referencias}

1.Almeida MFB, Kawakami MD, Moreira LMO, Santos RMVD, Anchieta LM, Guinsburg R. Early neonatal deaths associated with perinatal asphyxia in infants $\geq 2500 \mathrm{~g}$ in Brazil. J Pediatr (Rio J). 2017;93(6):576-84.

2.Daripa M, Caldas HM, Flores LP, Waldvogel BC, Guinsburg R, de Almeida MF. Perinatal asphyxia associated with early neonatal mortality: populational study of avoidable deaths. Rev Paul Pediatr. 2013;31(1):37-45.

3.Penela-Vélez de Guevara MT, GilLópez SB, Martín-Puerto MJ, RomeroEscós MD, Herrera-Martín M, UrbónArtero A. A descriptive study of perinatal asphyxia and its sequelae. Rev Neurol. 2006;43(1):3-6.

4.Secretaría Distrital de Salud. Lineamiento técnico para el manejo de la asfixia perinatal. Bogotá: Secretaría; 2015.

5.Shankaran S. Outcomes of hypoxicischemic encephalopathy in neonates treated with hypothermia. Clin Perinatol. 2014;41(1):149-59.

6.Volpe J. Neurología del recién nacido. 5 ed. Madrid: McGraw Hill; 2013.

7.Centro Nacional de Investigación en Evidencia. Guía de práctica clínica del recién nacido con asfixia perinatal. Bogotá; Ministerio de Salud; 2013.

8.Perlman JM, Wyllie J, Kattwinkel J, Atkins DL, Chameides L, Goldsmith JP, et al. Neonatal resuscitation: 2010 International Consensus on Cardiopulmonary Resuscitation and Emergency Cardiovascular Care Science with Treatment Recommendations. Pediatrics. 2010;126(5):e1319-44.

9.Jacobs SE, Berg M, Hunt R, Tarnow-Mordi WO, Inder TE, Davis PG. Cooling for newborns with hypoxic ischaemic encephalopathy. Cochrane Database Syst Rev. 2013(1):CD003311. https://doi.org/10. 1002/14651858.CD003311.pub3 
10.Shah PS. Hypothermia: A systematic review and meta-analysis of clinical trials. Semin Fetal Neonatal Med. 2010;15(5):238-46.

11.Manotas H, Troncoso G, Sánchez J, Molina G. Descripción de una cohorte de pacientes neonatos con diagnóstico de asfixia perinatal tratados con hipotermia terapéutica. Perinatol Reprod Hum. 2018;32(2):70-7.

12.Shankaran S, Pappas A, McDonald SA, Vohr BR, Hintz SR, Yolton $\mathrm{K}$, et al. Childhood outcomes after hypothermia for neonatal encephalopathy. $\mathrm{N}$ Engl J Med. 2012;366(22):2085-92.

13.Secretaría Distrital de Salud de Bogotá. Base de datos SDS y aplicativo Web RUAF_ND: datos Preliminares. Bogotá: Secretaría; 2017.

14.Mah-Mungyeh E, Chiabi A, Tchokoteu FL, Nguefack S, Bogne JB, Siyou HH, et al. Neonatal mortality in a referral hospital in Cameroon over a seven year period: Trends, associated factors and causes. Afr Health Sci. 2014;14(4):985-92.

15.Akinyemi JO, Bamgboye EA, Ayeni O. Trends in neonatal mortality in Nigeria and effects of bio-demographic and maternal characteristics. BMC Pediatr. 2015;15:36.

16.Wu QJ, Li LL, Li J, Zhou C, Huang YH. Time trends of neonatal mortality by causes of death in Shenyang. 1997-2014. Oncotarget. 2016;7 (13):16610-8.

17.Dallolio L, Lenzi J, Fantini MP. Temporal and geographical trends in infant, neonatal and post-neonatal mortality in Italy between 1991 and 2009. Ital J Pediatr. 2013;39:19.

18.Gregory EC, MacDorman MF, Martin JA. Trends in fetal and perinatal mortality in the United
States. 2006-2012. NCHS Data Brief. 2014(169):1-8.

19.Battin MR, Knight DB, Kuschel $\mathrm{CA}$, Howie RN. Improvement in mortality of very low birthweight infants and the changing pattern of neonatal mortality: the 50year experience of one perinatal centre. J Paediatr Child Health. 2012;48(7):596-9.

20.Shah V, Warre R, Lee SK. Quality improvement initiatives in neonatal intensive care unit networks: Achievements and challenges. Acad Pediatr. 2013;13(6 Suppl):S75-83.

21.Marantz P, Sáenz Tejeira MM, Peña G, Segovia A, Fustiñana C. Fetal and neonatal mortality in patients with isolated congenital heart diseases and heart conditions associated with extracardiac abnormalities. Arch Argent Pediatr. 2013;111(5):418-22.

22.Van Heerden C, Maree C, Janse van Rensburg ES. Strategies to sustain a quality improvement initiative in neonatal resuscitation. Afr J Prim Health Care Fam Med. 2016;8(2):e1e10.

23.Galvao TF, Silva MT, Marques MC, de Oliveira ND, Pereira MG. Hypothermia for perinatal brain hypoxia-ischemia in different resource settings: a systematic review. J Trop Pediatr. 2013;59(6):453-9.

24.Ranasinghe S, Or G, Wang EY, Ievins A, McLean MA, Niell CM, et al. Reduced cortical activity impairs development and plasticity after neonatal hypoxia ischemia. J Neurosci. 2015;35(34):11946-59.

25.Jose A, Matthai J, Paul S. Correlation of EEG, CT, and MRI brain with neurological outcome at 12 months in term newborns with hypoxic ischemic encephalopathy. J Clin Neonatol. 2013;2(3):125-30. 
26.Gluckman PD, Wyatt JS, Azzopardi D, Ballard R, Edwards AD, Ferriero $\mathrm{DM}$, et al. Selective head cooling with mild systemic hypothermia after neonatal encephalopathy: Multicentre randomised trial. Lancet. 2005;365(9460):663-70.

27.Wayock CP, Meserole RL, Saria $\mathrm{S}$, Jennings JM, Huisman TA, Northington FJ, et al. Perinatal risk factors for severe injury in neonates treated with whole-body hypothermia for encephalopathy. Am J Obstet Gynecol. 2014;211(1):41 e1-8.

28.Laptook A, Shanakaran S, Tyson J, et al. Effect of therapeutic hypothermia initiated afeter 6 hours of age on death or disability among newborns with hypoxicisquemic encephalopathy. JAMA. 2017;318(16):1530-60. 